\title{
Tunable structured illumination light sheet microscopy for background rejection and imaging depth in minimally processed tissues
}

Joseph R. Landry

Ryosuke Itoh

Jonathan M. Li

Stephen S. Hamann

Michael Mandella

Christopher H. Contag

Olav Solgaard 


\title{
Tunable structured illumination light sheet microscopy for background rejection and imaging depth in minimally processed tissues
}

\author{
Joseph R. Landry, ${ }^{a, *}$ Ryosuke Itoh, ${ }^{b}$ Jonathan M. Li, ${ }^{a}$ Stephen S. Hamann, ${ }^{a}$ Michael Mandella, ${ }^{c, d}$ \\ Christopher H. Contag, ${ }^{d}$ and Olav Solgaard ${ }^{a}$ \\ aStanford University, Edward L. Ginzton Laboratory, Stanford, California, United States \\ bSCREEN Holdings Co., Ltd., R\&D Department, Kyoto, Japan \\ 'Stanford University, Department of Radiology, Stanford, California, United States \\ ${ }^{d}$ Michigan State University, Institute for Quantitative Health Science and Engineering, Department of Biomedical Engineering, East Lansing, \\ Michigan, United States
}

\begin{abstract}
We demonstrate improved optical sectioning in light sheet fluorescence microscopy using tunable structured illumination (SI) frequencies to optimize image quality in scattering specimens. The SI patterns are generated coherently using a one-dimensional spatial light modulator for maximum pattern contrast, and the pattern spatial frequency is adjustable up to half the incoherent cutoff frequency of our detection objective. At this frequency, we demonstrate background reductions of 2 orders of magnitude. $\odot$ The Authors. Published by SPIE under a Creative Commons Attribution 4.0 Unported License. Distribution or reproduction of this work in whole or in part requires full attribution of the original publication, including its DOI. [DOI: 10.1117/1.JBO.24.4.046501]
\end{abstract}

Keywords: microscopy; spatial light modulators; image reconstruction.

Paper 180660R received Jan. 2, 2019; accepted for publication Mar. 22, 2019; published online Apr. 9, 2019.

\section{Introduction}

Light sheet fluorescence microscopy (LSFM) has transformed imaging in the life sciences with its ability to rapidly acquire optical sections in thick specimens. ${ }^{1}$ LSFM uses lower peak intensities than other optical sectioning techniques, leading to less photobleaching. In combination, the high speed and favorable photobleaching properties of LSFM make it a powerful volumetric reconstruction tool. However, LSFM has no inherent background rejection mechanism. When imaging in scattering samples, the light sheet broadens as it travels, leading to large out-of-focus components. Samples are often optically cleared to circumvent this problem, but high-quality hydrogel-based clearing techniques, such as CLARITY, typically require days to weeks for processing. ${ }^{2}$

To facilitate faster turnaround while suppressing scattered and out-of-focus background, we incorporate optical sectioning structured illumination microscopy (OS-SIM) into LSFM. Unlike super-resolution SIM, which reconstructs the final image from nine images and enhances lateral resolution, ${ }^{3}$ OS-SIM uses three phases and rejects background outside the focal plane. ${ }^{4}$ Originally, OS-SIM was used to acquire optical sections of thick specimens in wide-field microscopes. In LSFM, OS-SIM suppresses scattered and out-of-focus components of the light sheet as it broadens while traveling deeper into scattering specimens.

Previous demonstrations of OS-SIM in LSFM improved image quality using coherent and incoherently generated structured illumination (SI) patterns. However, they are limited in the highest achievable spatial frequency due to incoherent SI pattern generation ${ }^{5}$ or low power at high frequencies. ${ }^{6}$ Here, we introduce a microscope design that reconfigures the generation of the SI patterns using a spatial light modulator (SLM), enabling

*Address all correspondence to Joseph R. Landry, E-mail: jlandry@stanford .edu.edu high-contrast patterns up to the optimal sectioning frequency. ${ }^{7}$ These changes preserve image contrast at deeper imaging depths and allow tailoring of the SI pattern spatial frequency based on the scattering properties of the sample.

\section{Theory}

The light sheet microscope described here is built in the inverted open-top configuration (Fig. 1), enabling direct placement of samples onto the optical window. ${ }^{8}$ High-resolution imaging obliquely through the optical window is made possible using a hypohemispherical immersion lens. A layer of index matching fluid separates the fused silica window and hypohemisphere, allowing the window and sample to be scanned together.

The immersion lens improves image quality by removing the severe imaging aberrations of the optical window at the cost of introducing limited aberrations at the hypohemisphere-air interface. The lens is positioned such that the lens center of curvature coincides with the intersection of the illumination and detection optical axes. Rays arising from the center field point of the sample plane are normal to the hypohemisphere surface, and thus aberration-free, while field points off-axis are only slightly aberrated as rays intersect at the hypohemisphere-air interface at small angles. As in all immersion microscopes, the resolution and magnification are improved by a factor of $\frac{n_{x}}{n_{y}}$, with $n_{x}$ and $n_{y}$ referring to the index of the immersion and surrounding media, respectively.

In OS-SIM, the sample is illuminated with three identical sinusoidal intensity profiles, each with the same frequency but separated in phase by $\frac{2 \pi}{3}$. Features that lay within the objective depth-of-field are modulated according to the SI fringe pattern and kept in focus. Features above and below the focal plane are similarly modulated, but they are blurred according to the detection objective (DO) optical transfer function (OTF). Due to the blurring, the out-of-focus component of the image remains largely the same from one pattern to the next, despite the change 
(a)

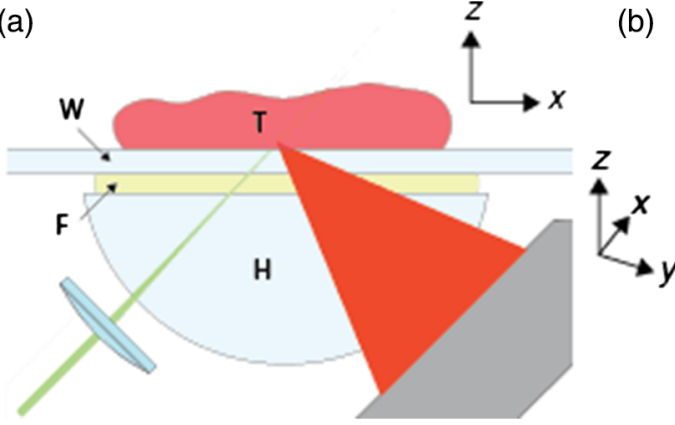

(b)

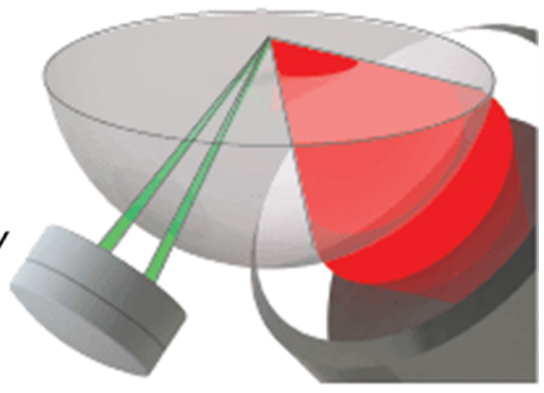

Fig. 1 (a) Sample interface cross section showing the tissue (T), optical window (W), matching fluid (F), and hypohemisphere $(H)$. Images acquired as the sample and window are translated along the $x$ direction. (b) Three-dimensional rendering of beams and hypohemisphere.

in phase. The out-of-focus component is then being removed using the following equation to reconstruct the image:

$I_{\mathrm{SI}}=\sqrt{\left(I_{1}-I_{2}\right)^{2}+\left(I_{2}-I_{3}\right)^{2}+\left(I_{3}-I_{1}\right)^{2}}$

where $I_{1}, I_{2}$, and $I_{3}$ refer to images captured with each successive SI phase. ${ }^{4}$ This equation rejects data that are the same between images (background), while preserving data that change (signal). To compare SI reconstructions with traditional light sheet images, we define the "sum" image, $I_{\text {sum }}=I_{1}+I_{2}+$ $I_{3}$, which closely approximates the same system without OS-SIM.

The OTF for OS-SIM in response to an infinitesimally thin fluorescent sheet is given as

$H(u, v) \approx g(v)\left\{2 \frac{J_{1}\left[u v\left(1-\frac{v}{2}\right)\right]}{u v\left(1-\frac{v}{2}\right)}\right\}$,

where $g(v)=1-0.69 v+0.0076 v^{2}+0.043 v^{3}$ and $J_{1}$ is the first Bessel function of the first kind. ${ }^{4}$ The normalized axial defocus coordinate $u$, which is the location of the fluorescent sheet relative to the DO, is given by $u=(8 \pi / \lambda) z \sin ^{2}(\alpha / 2)$, where $z$ is the real defocus coordinate, $\lambda$ is the wavelength, and $\alpha$ is the collection half-angle. The normalized spatial frequency of the SI pattern $v$ is given by $v=f \lambda /[n \sin (\alpha)]$, where $f$ is the real spatial frequency and $n$ is the immersion lens refractive index.

To quantify the optical sectioning strength at a given spatial frequency $v$, we translate the fluorescent sheet through the DO focus to compute the axial response of Eq. (2). We compute the full width at half maximum (FWHM) of the response to characterize the sectioning strength, which is found by solving for $u^{\prime}$ such that the OTF magnitude falls to half its original value: $\left|H\left(u^{\prime}, v\right)\right|=0.5|H(0, v)|$. The resulting FWHM, expressed as twice the real axial coordinate, $2 z$, is shown in Fig. 2 as a function of SI spatial frequency. Up to a point, high spatial frequency patterns are blurred more strongly outside the objective depthof-field, resulting in narrower optical sections. The narrowest sectioning occurs at $v=1$, after which further increases to the spatial frequency reduces the signal collected from the sample plane against an already blurred background, resulting in decreased sectioning power. The fraction of signal collected from the sample plane due to the SI spatial frequency modulation is given by $|H(0, v)|$. The signal-to-sectioning trade-off (SST), which is the normalized ratio of $|H(0, v)|$ to axial

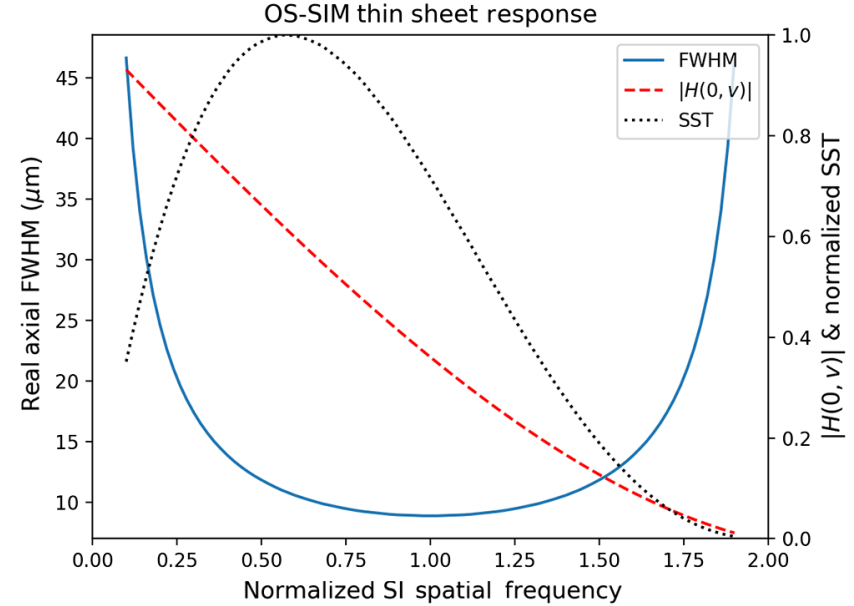

Fig. 2 Trade-off between narrower FWHM, corresponding to strong optical sectioning, and the signal, $|H(0, v)|$, which is the magnitude of the OTF, $H(u, v)$, at the sample plane $(u=0)$ at a particular SI spatial frequency, $v$. The SST curve is given by the normalized ratio of $|H(0, v)|$ to FWHM, peaks at $v=0.56$.

FWHM, guides optimization of the trade-off between sectioning strength and signal attenuation at varying spatial frequencies.

\section{Experimental Setup}

We generate our SI patterns using a grating light valve (GLV), which is a reflective, high-speed one-dimensional (1-D) SLM capable of switching at hundreds of $\mathrm{kHz} .{ }^{9,10}$ Conceptually, patterns with phases $0, \frac{2 \pi}{3}$, and $\frac{4 \pi}{3}$ are produced according to Fig. 3 . A square wave phase state is assigned to the GLV as periodic "on" and "off" pixels, thus creating a phase grating. On pixels have displacements of $\frac{\lambda}{4}$ relative to the off pixels for maximum first-order diffraction efficiency. A pair of slits in the screen (S) passes the two first orders, and after lens L2, the two beams interfere to create a sinusoidal field profile, the SI pattern, at plane $\mathrm{P}$, which is conjugate to the sample plane. To change the SI phase in equal increments of $\frac{2 \pi}{3}$, a periodic template consisting of three off and three on pixels is shifted by a single pixel from one state to the next for three total states [Fig. 3(b)].

Manipulation of the phase fixes the periodicity of the GLV, thus also fixing the spatial frequency of the SI interference pattern. However, the system of knife-edge prisms, mirrors, and relay lenses shown in Fig. 4 enable SI pattern frequency control. Translation of the second prism affects the beam separation, 
(a)

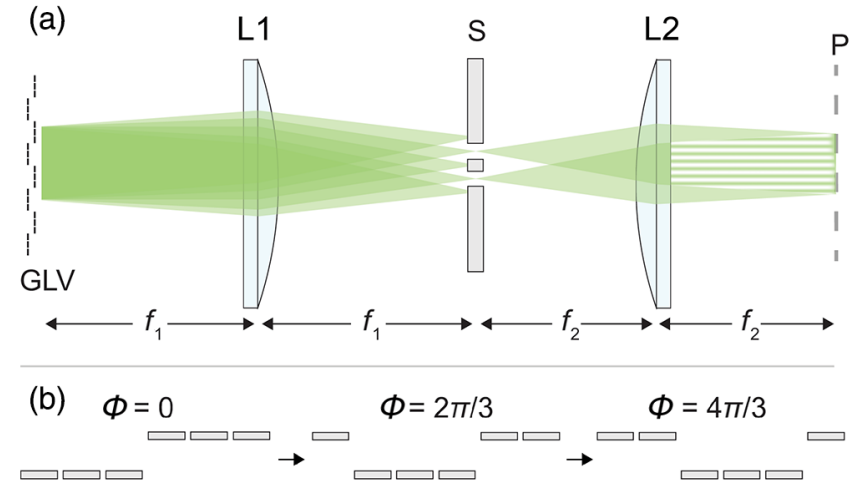

Fig. 3 (a) Generation of sinusoidal SI patterns by relaying the beam from the GLV to plane $P$ using lenses $L 1$ and $L 2$, passing only the first orders through double slit S. (b) Shifting the SI pattern phase $\phi$ by $\frac{2 \pi}{3}$ by shifting the repeating six-pixel template displayed on the GLV.

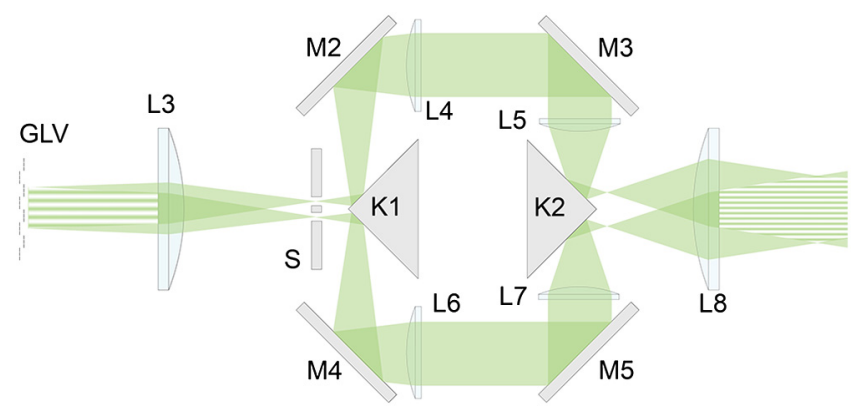

Fig. 4 Optical assembly for continuous SI pattern spatial frequency control. GLV, Grating light valve; L3 to L8, achromatic spherical lenses; S, double slit; K1 and K2, knife-edge prisms; M2 to M5, planar mirrors. The first knife-edge prism is imaged onto the second, which can be translated to pick the desired SI pattern spatial frequency.

thus altering the SI pattern spatial frequency at the sample plane. As a whole, this configuration generates three frequencyadjustable SI intensity patterns with phases $0, \frac{2 \pi}{3}$, and $\frac{4 \pi}{3}$. Unlike our previous system, ${ }^{6}$ the pattern power does not decrease with increasing pattern frequency. Furthermore, patterns are generated using shorter GLV pixel displacements $\left(\frac{\lambda}{4}\right.$ rather than $\left.\frac{\lambda}{3}\right)$, enabling the use of longer wavelengths.

The complete microscope is shown in Fig. 5. A 660-nm beam is expanded (L1 and L2). A cylindrical lens (C) focuses the beam onto the GLV (F1088-P, Silicon Light Machines). The diffracted orders pass through L3 to a pair of slits in screen S, which passes only the first-order beams. The beams are separated by a knife-edge prism, $\mathrm{K} 1$, and individually reduced in size (L4 to L7) as they are imaged onto a second knife-edge prism, K2, and passed through relay L8 and L9. After L9, the beams are rotated by 90 deg using a dove prism (D) before the beam reaches M6, which is conjugate to the rear pupil of illumination objective (IL). The beams are relayed (L10, L11, M7, and M8) through IL (AC127-025-B, Thorlabs, 25-mm focal length, 11.43-mm clear aperture), into the hypohemisphere (H) at 45 deg from the vertical axis. The focal length and clear aperture of IL are such that it provides the equivalent of $0.23 \mathrm{NA}$ ( 0.33 in the immersion lens) to create spatial frequencies up to one-half the detection NA. The individual NA of each of the incident beams in air is 0.031 . The fluorescent signal is collected by DO (MY20X-804, Mitutoyo, NA 0.42, 20×), at a right angle to IL, and subsequently focused by a tube lens (TL) and filtered

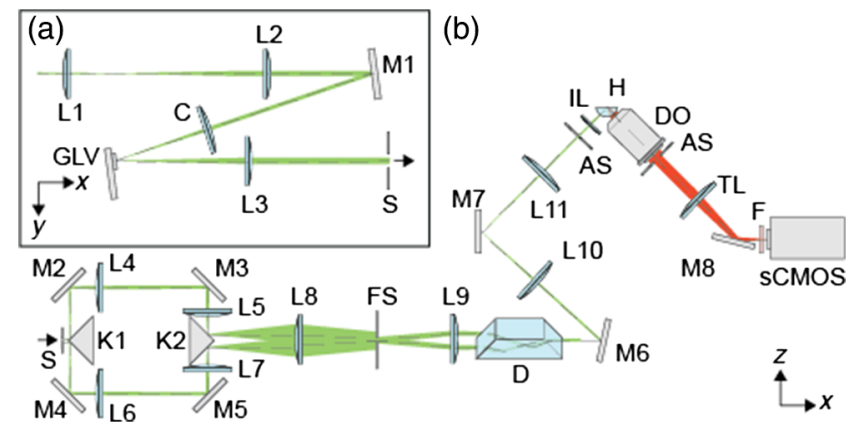

Fig. 5 System overview. L1 to L11, Achromatic spherical lenses; M1 to M8, planar mirrors; C, achromatic cylindrical lens; GLV, grating light valve; S, double slit; $\mathrm{K} 1$ and $\mathrm{K} 2$, knife-edge prisms; FS, field stop; $\mathrm{D}$, dove prism; AS, aperture stop; IL, illumination objective; DO, detection objective; TL, tube lens; $F$, emission filter. (a) Top view of system up to $S$ and (b) front view of system after $S$.
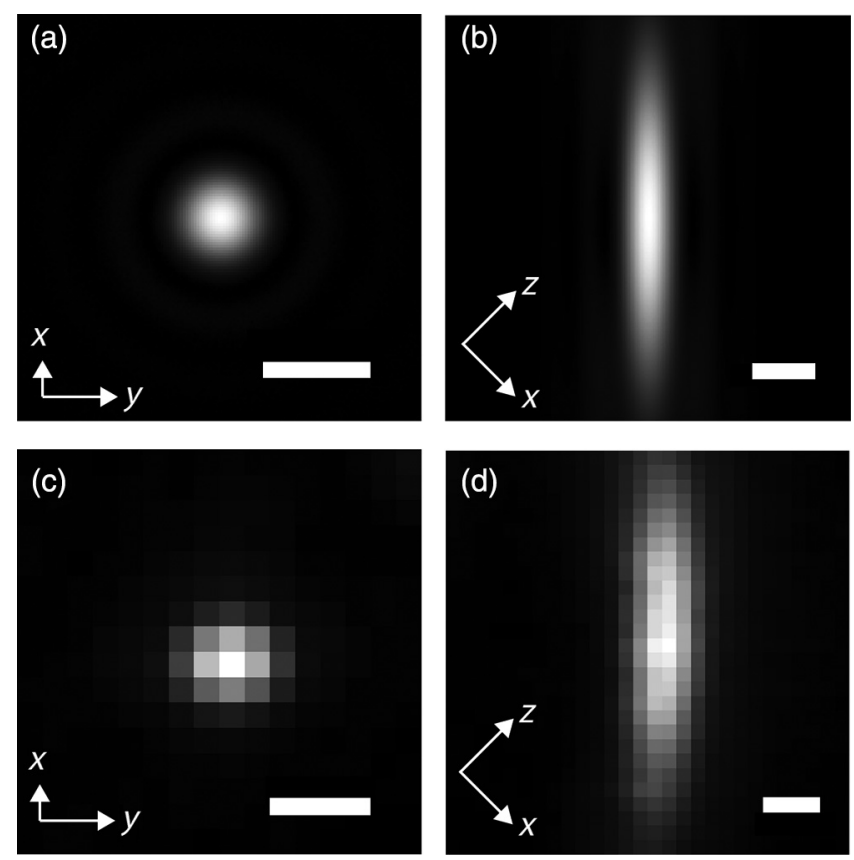

Fig. 6 Cross sections of the calculated and measured 3-D PSF along the detection axis. The lateral and axial sections are taken with respect to the detection axis, which is oriented 45 deg from the $x$ axis. (a) and (b) The calculated lateral and axial PSFs, with FWHM of 0.56 and $4.0 \mu \mathrm{m}$, respectively. Calculated PSF pixels are $0.025 \mu \mathrm{m}$. (c) and (d) The corresponding measured PSFs using 200-nm fluorescent sources, which have FWHM of 0.52 and $3.9 \mu \mathrm{m}$, respectively. Measured pixels are $0.23 \mu \mathrm{m}$. Scale bars in all plots are $1 \mu \mathrm{m}$.

(F) before the image forms on an sCMOS camera (Zyla 4.2PLUS).

The hypohemispherical lens increases illumination and collection NA by a factor of 1.45 , making the final illumination and collection NAs 0.045 and 0.61 , respectively. From the measured system magnification, 28.2, the pixel size in the object plane is $0.23 \mu \mathrm{m}$. Subresolution 200-nm fluorescent beads were used to measure the three-dimensional (3-D) point spread function (PSF) of the system [Figs. 6(c) and 6(d)]. The measured lateral and axial FWHM of the PSF, 0.52 and $3.9 \mu \mathrm{m}$, respectively, are in good agreement with the calculated values, 0.56 and $4.0 \mu \mathrm{m}$ [Figs. 6(a) and 6(b)]. The Gaussian beam waist of the light sheet is $5.1 \mu \mathrm{m}$ and the apodized width is $430 \mu \mathrm{m}$. The optimal 
sectioning thickness, occurring at normalized frequency 1.0 , is $5.3 \mu \mathrm{m}$.

\section{Results}

To compare background rejection at different pattern frequencies, we collected images of 200-nm dark red fluorescent beads

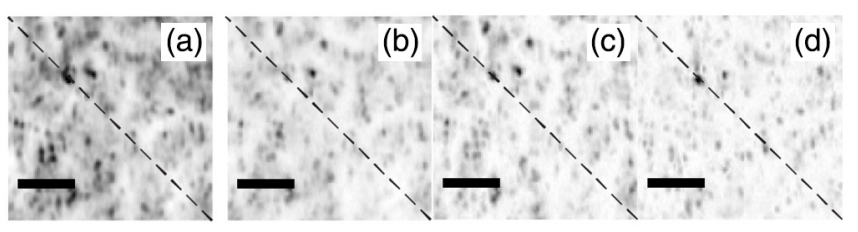

(e) Background rejection in scattering matrix

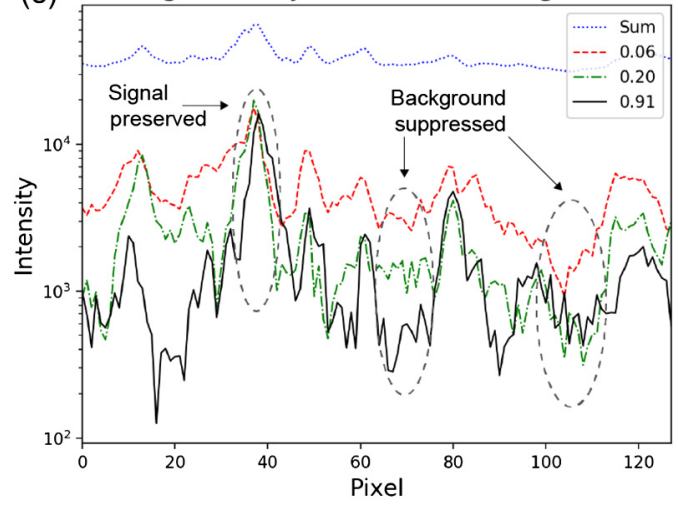

Fig. 7 Background rejection in a scattering bead phantom at different SI spatial frequencies. (a)-(d) Inverted $128 \times 128$ pixel regions from sections reconstructed parallel to the optical window. (a) The sum (non-SI) image, while (b)-(d) SI reconstructions with normalized spatial frequencies $0.06,0.20$, and 0.90 , respectively. Scale bars are $8 \mu \mathrm{m}$. (e) Cross sections along each dotted line of (a)-(d).
(ThermoFisher F8807) suspended in a scattering matrix (Fig. 7). The suspension is composed of water, 5\% Intralipid, and $1 \%$ agarose, which results in a matrix with a scattering coefficient of roughly $100 \mathrm{~cm}^{-1}$, similar to many human tissues. ${ }^{11}$ The strong scattering present in this sample results in large background levels in the vicinity of the fluorescent sources, obscuring the beads. To limit the effect of photobleaching on repeated scanning of the same volume, the power is kept low and frame rate limited to 75 frames per second. Improvements to bead contrast in reconstructed sections parallel to the optical window using three different SI spatial periods are shown in Figs. 7(a)-7(d). Each $128 \times 128$ pixel image is linearly scaled to saturate $0.05 \%$ of pixel values at 0 and 1 , and then inverted to clearly show the background levels. The true intensity values are compared in Fig. 7(e). Using SI, even with a low spatial frequency, reduces background by roughly an order of magnitude compared to the sum image. Background continues to be suppressed as the normalized SI frequency increases from 0.06 to 0.20 , and again from 0.20 to 0.91 , resulting in a total background reduction of 2 orders of magnitude relative to the sum image case.

To demonstrate our microscope in a practical setting, we imaged an excision of mouse colon tissue over a range of SI spatial frequencies (Fig. 8). The sample was first stained for $10 \mathrm{~min}$ in a phosphate-buffered solution (PBS) with $20 \mu \mathrm{M}$ DRAQ5, a dye which localizes nuclear features and is excited at $660 \mathrm{~nm}$. Next, the tissue was submerged in a waterthiodiethanol solution, of refractive index 1.46 , for $5 \mathrm{~min}$ to remove residual PBS and create optimal index matching between the sample and the glass window. Images with size $2048 \times 1400$ pixel were taken with a range of normalized SI spatial frequencies from 0.06 to 0.74 at 60 frames per second. Improvements to image contrast are seen in Figs. 9(a) through

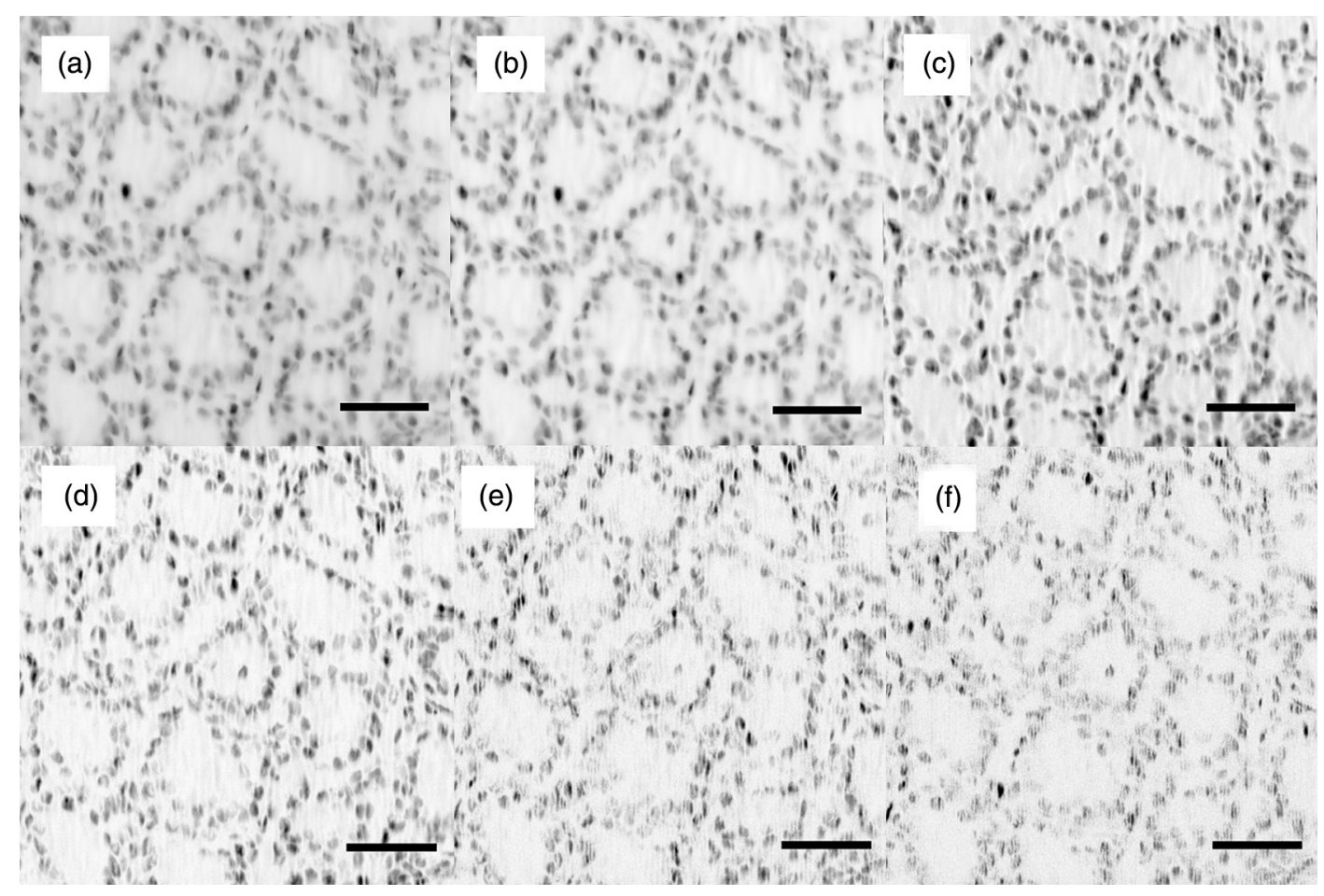

Fig. 8 Inverted images of mouse colon crypts stained with nuclear dye DRAQ5 at a depth of $100 \mu \mathrm{m}$. (a) Sum image and (b)-(f) SI images with normalized spatial frequencies $0.06,0.20,0.38,0.56$, and 0.74 , respectively. Scale bars are $40 \mu \mathrm{m}$. 


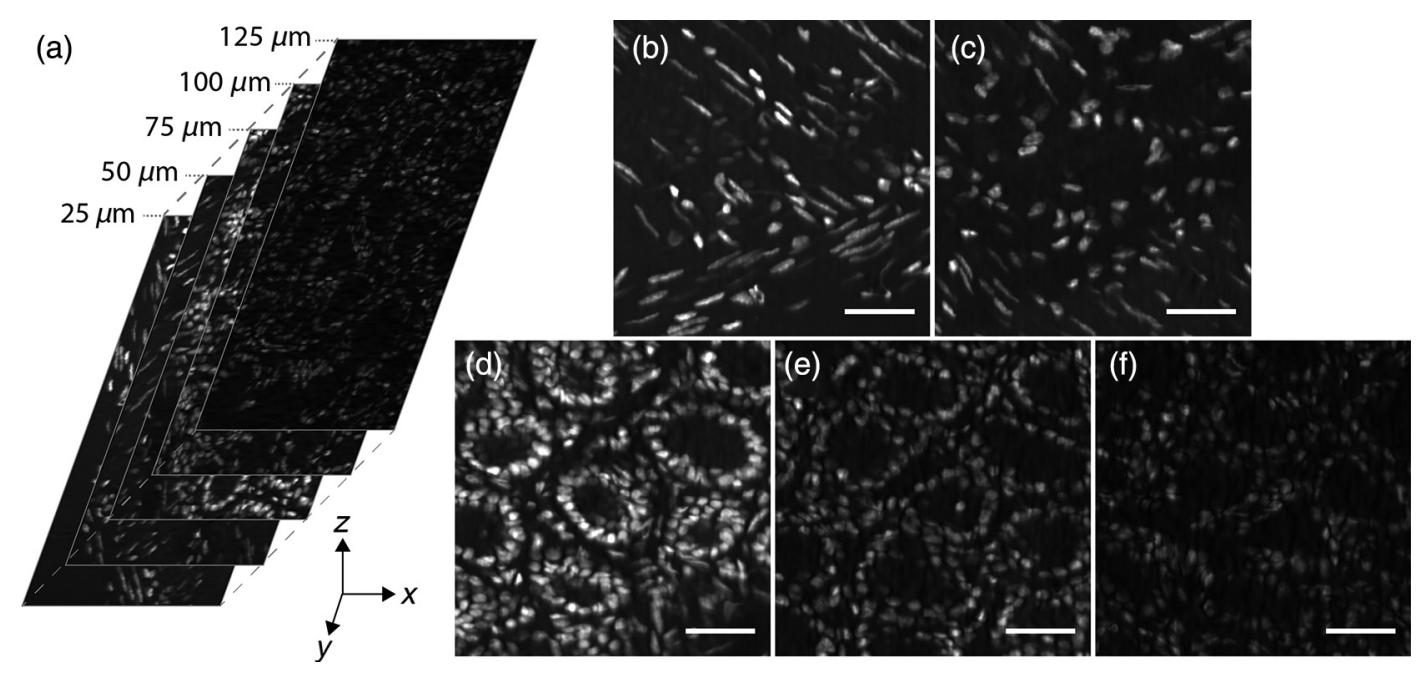

Fig. 9 Sections from 3-D mouse colon image with a fixed normalized SI pattern frequency of 0.38 . (a) Sections in original position with spacings of $25 \mu \mathrm{m}$ and (b)-(f) 25-, 50-, 75-, 100-, and 125- $\mu \mathrm{m}$ depths, respectively. (b) and (c) Shallow muscular layers and the (d)-(f) deeper mucosal layer are clearly shown. Scale bars are $30 \mu \mathrm{m}$.

9(d), after which striping artifacts and signal attenuation begin to affect the integrity of the image. Figure 9 shows the same sample at $25-\mu \mathrm{m}$ intervals with a fixed normalized SI pattern frequency of 0.38 .

Figure 8 demonstrates the usefulness of a tunable SI spatial frequency in practical volumetric imaging. At a depth of $100 \mu \mathrm{m}$, normalized frequencies of 0.20 to 0.38 [Figs. 8(c) and 8(d)] appear to optimize the trade-off between background and signal rejection in such a highly scattering specimen. Imaging at shallower depths, from which more signal is collected, a higher frequency may be more suitable for narrower sections. At greater imaging depths, the pattern frequency should be lowered both to increase signal and avoid the high-frequency imaging artifacts as shown in Figs. 8(e) and 8(f), which are caused by distortion of the SI patterns as they travel through the sample. Artifacts are especially apparent in high-frequency patterns because small spatial deviations incur large effective pattern phase changes. This invalidates the assumption of Eq. (1) that all three patterns are separated in phase by $\frac{2 \pi}{3}$, which results in artifacts. Such deviations accumulate as the light sheet travels deeper into the specimen, leading to a trade-off between SI pattern frequency and maximum imaging depth. Reconstruction algorithms that account for phase errors and exhibit better signal to noise properties than Eq. (1) may result in improved image quality. ${ }^{12}$ Such algorithms offer a more robust reconstruction, quantifying the exact phase of each SI pattern, and performing reconstruction in frequency space.

\section{Conclusion}

In summary, we designed, built, and characterized a light sheet microscope that improves practical usage and image quality in scattering samples. Compared to another recent frequency-tunable SIM microscope, ${ }^{13}$ our implementation sacrifices the ability to perform super-resolution SIM, but is simpler, requiring only a single objective, and faster, as the high-speed SLM is used for phase-shifting rather than a piezo stage. An alternative SI pattern generation scheme that maintains a common optical path layout, rather than splitting the beams, uses a twodimensional ferroelectric SLM in place of our 1-D SLM, which allows fine-tuning of the SI frequency, better phase stability, and requires no mechanical movement. ${ }^{14}$ An improved GLV that extends the maximum attainable phase modulation from $1.25 \pi$ to $2 \pi$ at $660 \mathrm{~nm}$ could also solve this problem using two linear phase ramps on a 1-D device, symmetric about the beam center. $^{15}$

The 13.3-kHz switching speed of the GLV is advantageous when imaging small camera sensor subregions, as it can keep up with state-of-the-art scientific CMOS cameras that achieve roughly 400 frames per second over a $512 \times 512$ pixel area and 1500 frames per second over a $128 \times 128$ pixel area. ${ }^{16}$ Multiple regions of varying depth, each with a different, optimized SI spatial frequency, could be sequentially scanned and combined, thus maintaining image quality throughout the specimen.

\section{Disclosures}

The authors declare no conflicts of interest.

\section{Acknowledgments}

This research was funded through National Institutes of Health (NIH) 5R01CA180152-02. The authors thank Silicon Light Machines for both providing the phase GLV and troubleshooting the operation of the modulator. We also thank Stephan Rogalla at the Gambhir Lab, Stanford University for providing biological tissues.

\section{References}

1. J. Huisken et al., "Optical sectioning deep inside live embryos by selective plane illumination microscopy," Science 305(5686), 1007-1009 (2004).

2. D. S. Richardson and J. W. Lichtman, "Clarifying tissue clearing," Cell 162(2), 246-257 (2015).

3. M. G. Gustafsson, "Surpassing the lateral resolution limit by a factor of two using structured illumination microscopy," J. Microsc. 198(2), 82-87 (2000).

4. M. Neil, R. Juškaitis, and T. Wilson, "Method of obtaining optical sectioning by using structured light in a conventional microscope," Opt. Lett. 22(24), 1905-1907 (1997).

5. P. J. Keller et al., "Fast, high-contrast imaging of animal development with scanned light sheet-based structured-illumination microscopy," Nat. Methods 7(8), 637-642 (2010). 
6. R. Itoh et al., "Light sheet fluorescence microscopy using high-speed structured and pivoting illumination," Opt. Lett. 41(21), 5015-5018 (2016).

7. D. Karadaglić and T. Wilson, "Image formation in structured illumination wide-field fluorescence microscopy," Micron 39(7), 808-818 (2008).

8. A. K. Glaser et al., "Light-sheet microscopy for slide-free nondestructive pathology of large clinical specimens," Nat. Biomed. Eng. 1(7), 0084 (2017).

9. O. Solgaard, F. Sandejas, and D. Bloom, "Deformable grating optical modulator," Opt. Lett. 17(9), 688-690 (1992).

10. A. Payne et al., "Enabling high-data-rate imaging applications with grating light valve technology," Proc. SPIE 5348, 76-88 (2004).

11. W.-F. Cheong, S. A. Prahl, and A. J. Welch, "A review of the optical properties of biological tissues," IEEE J. Quantum Electron. 26(12), 2166-2185 (1990).

12. K. Wicker et al., "Phase optimisation for structured illumination microscopy," Opt. Express 21(2), 2032-2049 (2013).

13. B.-J. Chang, V. D. Perez Meza, and E. H. K. Stelzer, "csiLSFM combines light-sheet fluorescence microscopy and coherent structured illumination for a lateral resolution below $100 \mathrm{~nm}$," Proc. Natl. Acad. Sci. U. S. A. 114(19), 4869-4874 (2017).
14. R. Fiolka et al., "Time-lapse two-color 3D imaging of live cells with doubled resolution using structured illumination," Proc. Natl. Acad. Sci. U. S. A. 109(14), 5311-5315 (2012).

15. C. Garbellotto and J. M. Taylor, "Multi-purpose SLM-light-sheet microscope," Biomed. Opt. Express 9, 5419-5436 (2018).

16. C. Coates, B. Fowler, and G. Holst, "Scientific CMOS technology a high-performance imaging breakthrough" (2009).

Joseph R. Landry is a $\mathrm{PhD}$ student at Stanford University. He received his $B S$ degree in engineering physics from the University of Pittsburgh in 2013 and his MS degree in electrical engineering from Stanford University in 2015. His current research interests include microscopy, phased arrays, and adaptive optics.

Olav Solgaard earned his PhD from Stanford University in 1992. His doctoral dissertation, "Integrated semiconductor light modulators for fiber-optic and display applications," was the basis for the establishment of a silicon valley firm Silicon Light Machines, cofounded by him in 1994. In 1999, he joined the Electrical Engineering Department, Stanford University. His research interests include optical MEMS, photonic crystals, optical sensors, electron optics, and solar energy conversion.

Biographies of the other authors are not available. 